\title{
Dynamic Certification and Reputation for Quality Online Appendix
}

\author{
Iván Marinovic, Andrzej Skrzypacz and Felipe Varas
}

\section{A Proofs of results in Section II}

\section{Proof of Lemma 1}

Proof. Let $p_{a} \equiv \sup \{p \in[0,1]: a(p)>0\}, p_{c} \equiv \sup \{p \in[0,1]: d(p, H)=1\}, \tau_{a} \equiv \inf \{t>$ $\left.0: p_{t}=p_{a}, p_{0}=1\right\}$, and $\tau_{c} \equiv \inf \left\{t>0: p_{t}=p_{c}, p_{0}=1\right\}$.

First, we show that in any equilibrium $p_{a} \leq p_{c}$. Looking for a contradiction, suppose that $p_{a}>p_{c}$. Let's consider the behavior of beliefs at the threshold $p_{a}$. If $a\left(p_{a}\right) \geq p_{a}$ then $\lambda\left(a\left(p_{a}\right)-p_{a}\right) \geq 0$ so beliefs never cross the threshold $p_{a}$. On the other hand, if $a\left(p_{a}\right)<p_{a}$ then beliefs cross the threshold $p_{a}$ however if this is the case, we have that $k / \lambda=D\left(p_{a}\right)=e^{-(r+\lambda)\left(\tau_{c}-\tau_{a}\right)} D\left(p_{c}\right)<e^{-(r+\lambda)\left(\tau_{c}-t\right)} D\left(p_{c}\right)=D\left(p_{t}\right)$ for all $t \in\left(\tau_{a}, \tau_{c}\right]$. This means that $a\left(p_{a}-\epsilon\right)=1$ but if this is the case then beliefs can never cross the threshold $p_{a}$. This in turn implies that $\tau_{c}=\infty$, so that $D\left(p_{t}\right)=e^{-(r+\lambda)\left(\tau_{c}-t\right)} D\left(p_{c}\right)=0$. This contradicts the hypothesis that $p_{a}>p_{c} \geq 0$ which requires that $\lambda D\left(p_{a}\right) \geq k$.

Second, we analyze the certification strategy. By definition we have that $d(p, \theta)=0$ for $p>p_{c}$ and $d\left(p_{c}, H\right)=1$. If the firm fails to certify at time $\tau_{c}$ beliefs drop to zero so $p_{\tau_{c}^{+}}=0$. The next step is to specify the certification strategy when $p_{0}=0$. We consider two cases: $V_{H}(1)-c>0$ and $V_{H}(1)-c=0\left(V_{H}(1)-c<0\right.$ is trivial because in this case certification is suboptimal so $\left.d_{t}=0\right)$. Let's consider the case with $V_{H}(1)-c>0$ first. Suppose that $\tilde{p}=\inf \{p: d(p, H)=1\}>0$ and let $\tilde{\tau}=\inf \left\{t: p_{t}=\tilde{p}, p_{0}=0\right\}$. Using the incentives equation we have

$$
D(0)=e^{-(r+\lambda) \tilde{\tau}} D(\tilde{p}) .
$$

By construction we have that $V_{H}(\tilde{p})=V_{H}(1)-c=V_{H}\left(p_{c}\right)=V_{H}(0)$ (Note that it cannot be the case that $V_{H}\left(p_{c}\right) \neq V_{H}(0)$ as this would contradict the optimality of the certification 
strategy). Similarly, we also have $V_{L}(\tilde{p})=V_{L}(0)$ because the market infers that the firm has low quality if it fails to certify when $p_{t}=\tilde{p}$. Thus, $D(\tilde{p})=V_{H}(\tilde{p})-V_{L}(\tilde{p})=V_{H}(0)-V_{L}(0)=$ $D(0)=D\left(p_{c}\right)$. Replacing this in the equation (7) we get

$$
D(0)=e^{-(r+\lambda) \tilde{\tau}} D(0) \Rightarrow D(0)=0 .
$$

If this is the case then we have that $a(p)=0$ for all $p \in[0, \tilde{p}]$ and in particular $a(0)=0$ so $\left(p_{0}=0, \theta_{0}=L\right)$ is an absorbing state and $V_{L}(0)=0$. This, together with $D(0)=0$, implies that $V_{H}(0)=0$, which contradicts the hypothesis $V_{H}(1)-c>0$. Hence, it must be the case that $\tilde{p} \leq 0$.

Next, we consider the case with $V_{H}(1)-c=0$. In this case, by a similar argument as the one used before, we have that $D(0)=0$, so $a(0)=0$ and $\left(p_{t}=0, \theta_{t}=L\right)$ is an absorbing

state. This means that for any strategy $\tilde{d}_{t}$ in which the low quality firm never certifies there is some threshold $p_{c}$ such that $\operatorname{Pr}\left(\tilde{d}_{t}=\mathbf{1}_{\left\{p_{t} \leq p_{c}, \theta_{t}=H\right\}} \mid \theta_{0}\right)=1$ for all $t \geq 0$. Moreover, the restriction to strategies in which the low type never certifies is without loss of generality as in equilibrium the low type would never find optimal to certify low quality.

\section{Proof of Proposition 1}

Proof. We need to analyze several cases depending on the cost of certification and whether we have investment in equilibrium or not. In absence of investment we have that quality starts at $\theta=H$, it depreciates at a rate $\lambda$ and $\theta=L$ is an absorbing state. The first set of results characterizes the value function when this is the case.

\section{Equilibria with No Investment}

In absence of investment, the only decision for the firm is when to disclose. If the value function is increasing in beliefs, then the certification strategy is characterized by a certification threshold $p_{c}$. Let $\tau$ be the first time beliefs reach the certification threshold $p_{c}$. Direct computation yields the value function which is given by

$$
\begin{aligned}
V_{L}\left(p_{t}\right) & =\int_{t}^{\tau} e^{-r(s-t)} p_{s} d s \\
V_{H}\left(p_{t}\right) & =\int_{t}^{\tau} e^{-r(s-t)} p_{s} d s+e^{-(r+\lambda)(\tau-t)}\left(V_{H}\left(p_{0}\right)-c\right) .
\end{aligned}
$$


The certification threshold $p_{c}$ is an equilibrium if and only if $V_{H}(p) \geq V_{H}\left(p_{0}\right)-c$ for all $p \geq p_{c}$ so the firm does not want to accelerate certification, and $V_{H}\left(p_{c}\right) \geq c$ so the firm's benefit of certification is higher than the cost.

Step 1: $V_{H}\left(p_{c}\right) \geq c$. Using (2) and $p_{t}=e^{-\lambda t} p_{0}=e^{-\lambda t}$ we get

$$
\begin{aligned}
V_{H}\left(p_{0}\right) & =\frac{\int_{0}^{\tau} e^{-r s} p_{s} d s}{1-e^{-(r+\lambda) \tau}}-\frac{e^{-(r+\lambda) \tau}}{1-e^{-(r+\lambda) \tau}} c \\
& =\frac{1}{r+\lambda}-\frac{e^{-(r+\lambda) \tau}}{1-e^{-(r+\lambda) \tau}} c
\end{aligned}
$$

which is an increasing function $\tau$ and so a decreasing function of $p_{c}$ ( $\tau$ is decreasing in the threshold). Moreover, $V_{H}\left(p_{0}\right) \rightarrow-\infty$ as $\tau \rightarrow 0$; hence, there is a threshold $p_{c}^{+}$such that $V_{H}\left(p_{0}\right)=c$. This means that $p_{c}$ can be an equilibrium certification threshold only if $p_{c} \leq p_{c}^{+}$. Moreover, $p_{c}^{+}>0$ if and only if $c<\frac{1}{r+\lambda}$; otherwise, the unique equilibrium has no certification.

Step 2: $V_{H}(p) \geq V_{H}\left(p_{0}\right)-c$ for all $p \geq p_{c}$. A necessary condition for this to be the case is that $V_{H}^{\prime}\left(p_{c}\right) \geq 0$; otherwise, there is $\epsilon$ such that $V_{H}\left(p_{c}+\epsilon\right)<V_{H}\left(p_{0}\right)-c$. If we differentiate (2) with respect to time we get

$$
\begin{aligned}
\frac{d}{d t} V_{H}\left(p_{t}\right) & =-p_{t}+r \int_{t}^{\tau} e^{-r(s-t)} p_{s} d s+(r+\lambda) e^{-(r+\lambda)(\tau-t)}\left(V_{H}\left(p_{0}\right)-c\right) \\
& =-p_{t}+r \int_{t}^{\tau} e^{-(r+\lambda)(s-t)} p_{t} d s+(r+\lambda) e^{-(r+\lambda)(\tau-t)}\left(\frac{1}{r+\lambda}-\frac{c}{1-e^{-(r+\lambda) \tau}}\right) \\
& =e^{-(r+\lambda)(\tau-t)}\left(1-\frac{r}{r+\lambda} p_{t}\right)-\frac{\lambda}{r+\lambda} p_{t}-\frac{c(r+\lambda) e^{-(r+\lambda)(\tau-t)}}{1-e^{-(r+\lambda) \tau}}
\end{aligned}
$$

Because $p_{t}$ is decreasing in $t$ we have that $V_{H}^{\prime}\left(p_{t}\right) \geq 0$ if and only if $\frac{d}{d t} V_{H}\left(p_{t}\right) \leq 0$. This is true at time $\tau$ if and only if

$$
\left.\frac{d}{d t} V_{H}\left(p_{t}\right)\right|_{t=\tau}=1-p_{\tau}-\frac{c(r+\lambda)}{1-e^{-(r+\lambda) \tau}} \leq 0
$$

Using $p_{\tau}=p_{c}$ and $\tau=-\log \left(p_{c}\right) / \lambda$ we get the condition

$$
1-p_{c}-\frac{c(r+\lambda)}{1-p_{c}^{\frac{r+\lambda}{\lambda}}} \leq 0
$$

The left hand side of equation (3) is decreasing in $p_{c}$. Hence, there is $p_{c}^{-}$such that (3) holds 
with equality if and only if $c \leq 1 /(r+\lambda)$. Moreover, if this is the case, then condition (3) holds for any $p_{c} \geq p_{c}^{-}$. Hence, $p_{c}^{-}$is a lower bound for the certification threshold.

This is only a necessary condition; we still have to verify that $V_{H}(p) \geq V_{H}\left(p_{0}\right)-c$ for $p>p_{c}$. Taking the second derivative of $V_{H}\left(p_{t}\right)$ we get

$$
\begin{aligned}
\frac{d^{2}}{d t^{2}} V_{H}\left(p_{t}\right) & =(r+\lambda) e^{-(r+\lambda)(\tau-t)}\left(1-\frac{r}{r+\lambda} p_{t}-\frac{c(r+\lambda)}{1-e^{-(r+\lambda) \tau}}\right) \\
& -\left(e^{-(r+\lambda)(\tau-t)} \frac{r}{r+\lambda}+\frac{\lambda}{r+\lambda}\right) \dot{p}_{t} \\
& =(r+\lambda)\left(\frac{d}{d t} V_{H}\left(p_{t}\right)+\frac{\lambda}{r+\lambda} p_{t}\right)-\left(e^{-(r+\lambda)(\tau-t)} \frac{r}{r+\lambda}+\frac{\lambda}{r+\lambda}\right) \dot{p}_{t}
\end{aligned}
$$

Hence, we have that $\frac{d}{d t} V_{H}\left(p_{t}\right)=0$ implies $\frac{d^{2}}{d t^{2}} V_{H}\left(p_{t}\right)>0$. This means that if at time $\tau$ we have $\frac{d}{d t} V_{H}\left(p_{t}\right) \leq 0$ then it must be true that $\frac{d}{d t} V_{H}\left(p_{t}\right) \leq 0$ for all $t<\tau$. Thus, we have that

$$
V_{H}\left(p_{\tau}\right)-V_{H}\left(p_{t}\right)=\int_{t}^{\tau} \frac{d}{d t} V_{H}\left(p_{s}\right) d s \leq 0
$$

so $V_{H}\left(p_{t}\right) \geq V_{H}\left(p_{\tau}\right)=V_{H}\left(p_{0}\right)-c$. The final step is to see in which situations the equilibrium has no investment.

\section{Step 3: Investment Incentives}

We can compute the incentives to invest using equations (1) and (2)

$$
D\left(p_{t}\right)=e^{-(r+\lambda)(\tau-t)}\left(V_{H}\left(p_{0}\right)-c\right)=e^{-(r+\lambda)(\tau-t)}\left(\frac{1}{r+\lambda}-\frac{c}{1-e^{-(r+\lambda) \tau}}\right) .
$$

Hence, $D\left(p_{t}\right)<\frac{k}{\lambda}$ for all $t \leq \tau$ if and only if

$$
\frac{1}{r+\lambda}-\frac{c}{1-e^{-(r+\lambda) \tau}}<\frac{k}{\lambda}
$$

This condition is true for any $\tau$ if and only if $\frac{1}{r+\lambda}-c<\frac{k}{\lambda}$. Otherwise, this is true if and only if

$$
\tau<-\frac{1}{r+\lambda} \log \left(1-\frac{c}{\frac{1}{r+\lambda}-\frac{k}{\lambda}}\right)
$$

which corresponds to the certification time $\tau$ consistent with the threshold $p_{c}$ in the first part of Proposition 1. 


\section{Equilibria with Investment}

We have already characterized the equilibria that have no investment. The final step is to look at those equilibria in which there is positive investment. The boundary conditions at $p_{c}$ are given by

$$
\begin{aligned}
V_{H}\left(p_{c}\right)=V_{H}(0) & =V_{H}(1)-c \\
V_{L}\left(p_{c}\right)=V_{L}(0) & =\frac{\lambda a\left(V_{H}(1)-c\right)-a k}{r+\lambda a}
\end{aligned}
$$

Equation (4) can be rewritten

$$
V_{H}(0)=\left(\frac{r}{\lambda a}+1\right) V_{L}(0)+\frac{k}{\lambda}
$$

hence

$$
D(0)=\frac{r V_{L}(0)}{\lambda a}+\frac{k}{\lambda}
$$

On the other hand, $t \rightarrow D\left(p_{t}\right)$ is a continuous function so in equilibrium we must have that

$$
D\left(p_{c}\right)=D(0)=\frac{k}{\lambda}
$$

Otherwise, the firm would invest when beliefs are just above $p_{c}$. We can thus conclude that

$$
V_{L}(0)=V_{L}\left(p_{c}\right)=0
$$

This in turn implies that

$$
V_{H}(1)=\frac{k}{\lambda}+c
$$

Let $\tau=\inf \left\{p_{t}: p_{\tau}=p_{c}\right\}$. In equilibrium, $a\left(p_{t}\right)=0$ implies that for $p_{t}>p_{c}$ we have

$$
\tau=-\frac{\log p_{c}}{\lambda}
$$

The value function for the high type is given by

$$
V_{H}\left(p_{t}\right)=\int_{t}^{\tau} e^{-r(s-t)} p_{s}+e^{-(r+\lambda)(\tau-t)}\left(V_{H}(1)-c\right) d s
$$


Using $p_{s}=e^{-\lambda(s-t)} p_{t}$ and $V_{H}(1)-c=k / \lambda$ we get

$$
V_{H}\left(p_{t}\right)=\frac{p_{t}}{r+\lambda}\left[1-\left(\frac{p_{c}}{p_{t}}\right)^{\frac{r+\lambda}{\lambda}}\right]+\frac{k}{\lambda}\left(\frac{p_{c}}{p_{t}}\right)^{\frac{r+\lambda}{\lambda}} .
$$

Similarly,

$$
V_{L}\left(p_{t}\right)=\frac{p_{t}}{r+\lambda}\left[1-\left(\frac{p_{c}}{p_{t}}\right)^{\frac{r+\lambda}{\lambda}}\right] .
$$

Now, we can compute $p_{c}$ using the condition $V_{H}(1)=c+k / \lambda$ which gives us

$$
\left(\frac{1}{r+\lambda}-\frac{k}{\lambda}\right)\left[1-p_{c}^{\frac{r+\lambda}{\lambda}}\right]=c
$$

SO

$$
p_{c}=\left[1-\frac{c}{\frac{1}{r+\lambda}-\frac{k}{\lambda}}\right]^{\frac{\lambda}{r+\lambda}} .
$$

Intuitively, $p_{c}$ decreases in $c$ and $k$. An equilibrium with certification and investment exists iff

$$
\frac{1}{r+\lambda}-\frac{k}{\lambda}>c
$$

Finally, no certification and no investment is an equilibrium if and only if

$$
V_{H}^{n c}(0)>V_{H}^{n c}(1)-c
$$

which means that

$$
c>\frac{1}{r+\lambda}
$$

\section{B Proofs of results in Section III}

\section{Proof of Lemma 2}

Proof. For further reference, $x \equiv e^{-r \tau}, y \equiv e^{-r \tau_{a}}, \alpha \equiv(r+\lambda) / r$ and $q \equiv 1-\beta$ (and we simplify notation by not pointing out which $\theta_{0}$ they correspond to since this is implied by the two cases we solve in sequence).

We start considering the case $\theta_{0}=H$. The payoff of the high quality firm given an 
arbitrary tuple $(x, y, q)$ is

$$
\begin{aligned}
\mathcal{U}_{H}(x, y, q) & =\frac{1}{r+\lambda}+\frac{y-x}{r}-\frac{x^{\alpha}}{r+\lambda}+\frac{x^{\alpha} y^{1-\alpha}-y}{r+\lambda}-(y-x) \frac{k}{r}+x\left(1-\left(\frac{x}{y}\right)^{\alpha-1}+x^{\alpha-1}\right)\left(\bar{U}_{H}-c\right) \\
& +x\left(\left(\frac{x}{y}\right)^{\alpha-1}-x^{\alpha-1}\right) q \bar{U}_{L} .
\end{aligned}
$$

and the incentive compatibility constraint in terms of $x, y, q$ is

$$
y^{\alpha}=x^{\alpha}\left(\frac{\lambda\left(\bar{U}_{H}-c-q \bar{U}_{L}\right)}{k}\right) .
$$

For a fixed investment threshold $\tau_{a}$, pinned down by $y$, we look for the optimal combination $(x, q)$ that implements this $y$. Using the binding incentive compatibility constraint we get that for the fixed $y$

$$
q^{\prime}(x)=\frac{\alpha}{x} \frac{\bar{U}_{H}-c-q \bar{U}_{L}}{\bar{U}_{L}} .
$$

The first derivative of $\mathcal{U}_{H}(x, y, q(x))$ with respect to $x$

$$
\frac{\partial \mathcal{U}_{H}(x, y, q(x))}{\partial x}=-\left[\frac{1-k}{r}-\left(\bar{U}_{H}-c\right)\right]-\frac{1}{r+\lambda}\left[y-\alpha x^{\alpha-1}\left(y^{1-\alpha}-1\right)\right]
$$

so the second derivative is

$$
\frac{\partial^{2} \mathcal{U}_{H}(x, y, q(x))}{\partial x^{2}}=\frac{1}{r+\lambda} \alpha(\alpha-1) x^{\alpha-2}\left(y^{1-\alpha}-1\right)>0 .
$$

so $\mathcal{U}_{H}(x, y, q(x))$ is convex (by definition $y<1$ and $\alpha>1$ ) in $x$. This means that for an arbitrary $y$, the best pair $(x, \beta)$ implementing $y$ is an extreme point which, since $q(x)$ is increasing, means that we only need to consider $q=0$ and $q=1$.

The proof for the case $\theta_{0}=L$ is analogous, with the minor difference that we can focus on $y=0$ since we know it is optimal (as we argued in the text in a way independent of this lemma). The expected payoff of the firm is

$$
\mathcal{U}_{L}(x, q)=\frac{(1-x)(1-k)}{r}+\frac{x^{\alpha}-1}{r+\lambda}+x\left[1-x^{\alpha-1}\right]\left(\bar{U}_{H}-c\right)+x^{\alpha} q \bar{U}_{L}
$$

In the best equilibrium, the incentive compatibility constraint binds at $t=0$ (since otherwise 
we could increase $\tau$ to save certification costs), so

$$
1=x^{\alpha}\left(\frac{\lambda\left(\bar{U}_{H}-c-q \bar{U}_{L}\right)}{k}\right),
$$

or:

$$
x^{\alpha} q \bar{U}_{L}=x^{\alpha}\left(\bar{U}_{H}-c\right)-\frac{k}{\lambda} .
$$

Therefore, a $q$ that satisfies the incentive compatibility constraint at $t=0$ is increasing in $x$ (intuitively, larger $x$ means smaller $\tau$ so less time till certification, so the equilibrium can be more lenient without removing incentives for investment). Substituting $q$ from this condition into $\mathcal{U}_{L}(x, q)$ we get:

$$
\mathcal{U}_{L}(x, q(x))=\frac{1-k}{r}-\frac{1}{r+\lambda}-\frac{k}{\lambda}-x\left(\frac{1-k}{r}-\left(\bar{U}_{H}-c\right)\right)+\frac{x^{\alpha}}{r+\lambda}
$$

The second derivative is

$$
\frac{d^{2}}{d x^{2}} \mathcal{U}(x, q(x))=\frac{\alpha(\alpha-1) x^{\alpha-2}}{r+\lambda}>0,
$$

this means that the expected payoff is convex in $x$ so the optimal $q$ is again either zero or one.

\section{Proof of Proposition 2}

The proof of Proposition 2 follows the following steps:

- For $\theta_{0}=H$

(i) First, we show that if $\bar{U}_{H} \geq 1 /(r+\lambda)-c$ then the best equilibrium given $\beta_{H}=1$ has full investment (Lemma 1) and $\tau_{H}^{*}=\tau_{H}^{F I}$.

(ii) Then we show that if $c$ is small then the best equilibrium given $\beta_{H}=1$ dominates the best equilibrium with $\beta_{H}=0$ (Lemma 2),

(iii) and a solution to the equation $e^{-(r+\lambda) \tau_{H}^{F I}}\left(U_{H}^{F I}\left(\tau_{H}^{F I}\right)-c\right)=k / \lambda$ satisfying $U_{H}^{F I}\left(\tau_{H}^{F I}\right)-c \geq 1 /(r+\lambda)$ exists (Lemma 3 ).

(iv) We conclude from the previous steps that for small $c, \tau_{H}^{*}=\tau_{H}^{F I}$ and $\bar{U}_{H}=$ $U_{H}^{F I}\left(\tau_{H}^{F I}\right)$ 
- For $\theta_{0}=L$

(i) First, we show that a solution $\hat{U}_{L}^{0}$ to equation (19) exists (Lemma 4),

(ii) and then show that $\beta_{L}=0$ is optimal when $c$ is small (Lemma 5).

- Next, we show that a high quality firm has incentives to certify at time $\tau_{\theta}^{*}$ (Lemma 6).

- Finally, we show that $\beta_{L}^{*}$ is non-decreasing in $c$ (Lemma 7).

For reference, throughout the proofs we use notation $x \equiv e^{-r \tau}$ and $y \equiv e^{-r \tau_{a}}, q \equiv 1-\beta$ and $\alpha \equiv(r+\lambda) / r$, and we omit the reference to $\theta_{0}$ since it is implied by the case described in each step.

Lemma 1. Suppose $\bar{U}_{H}-c \geq 1 /(r+\lambda)$ and $\beta_{H}^{*}=1$. Then in the equilibrium that achieves $\bar{U}_{H}, \tau_{H}^{*}=\tau_{H}^{F I}$ and $\tau_{a}=0$.

Proof. Consider $\theta_{0}=H, p_{0}=1$.

The incentive compatibility constraint that determines optimal investment policy can be written as:

$\tau_{a}(\tau)=\inf \left\{t_{a} \in[0, \tau]: e^{-(r+\lambda)\left(\tau-t_{a}\right)}\left(\bar{U}_{H}-c\right) \geq \frac{k}{\lambda}\right\}=\max \left\{0, \tau-\frac{1}{r+\lambda} \log \left(\frac{\lambda\left(\bar{U}_{H}-c\right)}{k}\right)\right\}$.

Let

$$
\mathcal{U}_{H}\left(\tau, \tau_{a}(\tau), 1\right)=\int_{0}^{\tau} e^{-r t}\left(p_{t}-\mathbf{1}_{t \geq \tau_{a}(\tau)} k\right) d t+e^{-r \tau} p_{\tau}\left(\bar{U}_{H}-c\right)
$$

denote the equilibrium payoff for a given $\tau$ and for $\beta_{H}=1$.

The best equilibrium for $\beta_{H}=1$ implements full investment if

$$
\tau_{H}^{F I} \in \arg \max _{\tau} \mathcal{U}_{H}\left(\tau, \tau_{a}(\tau), 1\right)
$$

Computing each individual term we get

$$
\begin{aligned}
\mathcal{U}_{H}\left(\tau, \tau_{a}, 1\right) & =\frac{1}{r+\lambda}+\frac{e^{-r \tau_{a}}-e^{-r \tau}}{r}-\frac{e^{-(r+\lambda) \tau}}{r+\lambda}+e^{-r \tau_{a}} \frac{e^{-(r+\lambda)\left(\tau-\tau_{a}\right)}-1}{r+\lambda}-\left(e^{-r \tau_{a}}-e^{-r \tau}\right) \frac{k}{r} \\
& +e^{-r \tau}\left(1-e^{-\lambda\left(\tau-\tau_{a}\right)}\left(1-e^{-\lambda \tau_{a}}\right)\right)\left(\bar{U}_{H}-c\right)
\end{aligned}
$$

This expression is not convex in $\left(\tau, \tau_{a}\right)$; for this reason, it is convenient to work with the transformed variables $x \equiv e^{-r \tau}$ and $y \equiv e^{-r \tau_{a}}$. Letting $\alpha \equiv(r+\lambda) / r$, we can write the 
payoff $\mathcal{U}_{H}\left(\tau, \tau_{a}, 1\right)$ as a function of the new variables (abusing notation for $\mathcal{U}$ ) as:

$\mathcal{U}_{H}(x, y)=\frac{1}{r+\lambda}+\frac{y-x}{r}-\frac{x^{\alpha}}{r+\lambda}+\frac{x^{\alpha} y^{1-\alpha}-y}{r+\lambda}-(y-x) \frac{k}{r}+x\left(1-\left(\frac{x}{y}\right)^{\alpha-1}+x^{\alpha-1}\right)\left(\bar{U}_{H}-c\right)$

Let $x^{*} \equiv e^{-r \tau_{H}^{F I}}$. For $x \in\left[x^{*}, 1\right]$ we argued in the text that $\tau_{a}=0$ and $x=x^{*}$ in this range is optimal. For any larger $x$, we do not get full investment, so $\tau_{a}>0$ and the incentive compatibility constraint can be written in terms of $x$ and $y$ as

$$
y=x \underbrace{\left(\frac{\lambda\left(\bar{U}_{H}-c\right)}{k}\right)^{\frac{1}{\alpha}}}_{M} .
$$

Hence, for $x \geq x^{*}$, letting $\mathcal{U}_{H}(x) \equiv \mathcal{U}_{H}(x, y(x))$, where $y(x)=M x$, we get:

$\mathcal{U}_{H}(x)=\frac{1}{r+\lambda}+\frac{(M-1)(x-k)}{r}+\frac{\left(M^{1-\alpha}-M\right) x}{r+\lambda}+x\left(1-M^{1-\alpha}\right)\left(\bar{U}_{H}-c\right)+x^{\alpha}\left(\bar{U}_{H}-c-\frac{1}{r+\lambda}\right)$

From here we get,

$$
\mathcal{U}_{H}^{\prime \prime}(x)=\alpha(\alpha-1) x^{\alpha-2}\left(\bar{U}_{H}-c-\frac{1}{r+\lambda}\right)
$$

So if $\bar{U}_{H}-c>\frac{1}{r+\lambda}$, then $\mathcal{U}_{H}(x)$ is convex. It implies that the maximum of $\mathcal{U}_{H}(x)$ is attained at an extreme point belonging to $\left\{0, x^{*}\right\}$. Finally, since

$$
\begin{aligned}
\mathcal{U}_{H}(0) & =\frac{1}{r+\lambda} \\
\mathcal{U}_{H}\left(x^{*}\right) & =\left(1-x^{*}\right) \frac{1-k}{r}+x^{*}\left(\bar{U}_{H}-c\right)
\end{aligned}
$$

we get that, if $\bar{U}_{H}-c>\frac{1}{r+\lambda}$, then $x=x^{*}=e^{-r \tau_{H}^{F I}}$ is optimal. As a corollary, since $\bar{U}_{H} \geq U_{H}^{F I}\left(\tau_{H}^{F I}\right)$, full investment is optimal for $\beta_{H}=1$ whenever $U_{H}^{F I}\left(\tau_{H}^{F I}\right)-c>\frac{1}{r+\lambda}$.

Lemma 2. There is $\tilde{c}_{1}>0$ such that for any $c \leq \tilde{c}_{1}$ the payoff in the best equilibrium with $\beta_{H}=1$ is higher than the highest payoff when $\beta_{H}=0$.

Proof. We can write the firm payoff as a function of $(x, y, q)$ as (again abusing notation for $\mathcal{U})$ : 


$$
\begin{aligned}
\mathcal{U}_{H}(x, y, q) & =\frac{1}{r+\lambda}+\frac{y-x}{r}-\frac{x^{\alpha}}{r+\lambda}+\frac{x^{\alpha} y^{1-\alpha}-y}{r+\lambda}-(y-x) \frac{k}{r}+x\left(1-\left(\frac{x}{y}\right)^{\alpha-1}+x^{\alpha-1}\right)\left(\bar{U}_{H}-c\right) \\
& +x\left(\left(\frac{x}{y}\right)^{\alpha-1}-x^{\alpha-1}\right) q \bar{U}_{L} .
\end{aligned}
$$

From the incentive compatibility constraint we have that

$$
q \bar{U}_{L}=\left(\bar{U}_{H}-c\right)-\frac{k}{\lambda}\left(\frac{y}{x}\right)^{\alpha}
$$

which can be replaced in the firm's payoff to get

$$
\begin{aligned}
\mathcal{U}_{H}(x, y) & =\frac{1}{r+\lambda}+\frac{y-x}{r}-\frac{x^{\alpha}}{r+\lambda}+\frac{x^{\alpha} y^{1-\alpha}-y}{r+\lambda}-(y-x) \frac{k}{r}+x\left(\bar{U}_{H}-c\right) \\
& -x\left(\left(\frac{x}{y}\right)^{\alpha-1}-x^{\alpha-1}\right)\left(\frac{y}{x}\right)^{\alpha} \frac{k}{\lambda} .
\end{aligned}
$$

Writing the incentive compatibility constraint for $q=1$ as

$$
y=x\left(\frac{\lambda\left(\bar{U}_{H}-c-\bar{U}_{L}\right)}{k}\right)^{\frac{1}{\alpha}}=x M
$$

and substituting $y(x)=x M$ to $\mathcal{U}_{H}(x) \equiv \mathcal{U}_{H}(x, y(x))$ we get:

$\mathcal{U}_{H}(x)=\frac{1}{r+\lambda}+\frac{(M-1)(1-k) x}{r}-\frac{x^{\alpha}}{r+\lambda}+x \frac{M^{1-\alpha}-M}{r+\lambda}+x\left(\bar{U}_{H}-c\right)-x\left(M^{1-\alpha}-x^{\alpha-1}\right) M^{\alpha} \frac{k}{\lambda}$

Differentiating with respect to $x$ we get that

$$
\begin{aligned}
\mathcal{U}_{H}^{\prime}(x) & =\frac{(M-1)(1-k)}{r}-\frac{\alpha x^{\alpha-1}}{r+\lambda}+\frac{M^{1-\alpha}-M}{r+\lambda}+\left(\bar{U}_{H}-c\right) \\
& -\left(M^{1-\alpha}-\alpha x^{\alpha-1}\right) M^{\alpha} \frac{k}{\lambda} \\
\mathcal{U}_{H}^{\prime \prime}(x) & =(\alpha-1) \alpha x^{\alpha-2}\left(\bar{U}_{H}-c-\bar{U}_{L}-\frac{1}{r+\lambda}\right)
\end{aligned}
$$

We need to consider two cases: $\bar{U}_{H}-c-\bar{U}_{L}-\frac{1}{r+\lambda}>0$ and $\bar{U}_{H}-c-\bar{U}_{L}-\frac{1}{r+\lambda} \leq 0$. In the first case, the payoff (given $q=1$ ) is convex and so full investment is optimal (by the 
same reasoning as in the proof of Lemma 1). Moreover, with full investment it is optimal to set $q=0$ as because this minimizes the certification cost. Let's assume then that that $\bar{U}_{H}-c-\bar{U}_{L}-\frac{1}{r+\lambda} \leq 0$. Let $x_{1}$ be the optimal $x$ when $q=1$. It must be the case that $x \in\left[0, M^{-1}\right]$ as any $x>M^{-1}$ implements the same investment as $M^{-1}$ but at a higher certification cost. Under the assumption that $\bar{U}_{H}-c-\bar{U}_{L}-\frac{1}{r+\lambda} \leq 0$ the function $\mathcal{U}_{H}(x)$ is concave and so a necessary and sufficient condition for $x_{1}=M^{-1}$ (so there is full investment, $\left.y_{1}=1\right)$ is that $\mathcal{U}_{H}^{\prime}\left(M^{-1}\right) \geq 0$. We can compute:

$$
\begin{aligned}
\mathcal{U}_{H}^{\prime}\left(M^{-1}\right) & =\frac{(M-1)(1-k)}{r}-\frac{M}{r+\lambda}+\left(\bar{U}_{H}-c\right)-(\alpha-1)\left(M^{1-\alpha} \frac{1}{r+\lambda}-M \frac{k}{\lambda}\right) \\
& =\frac{(M-1)(1-k)}{r}+\left(\bar{U}_{H}-c\right)-\frac{M}{r+\lambda}-\frac{M}{r}\left(\frac{k}{\bar{U}_{H}-c-\bar{U}_{L}} \frac{1}{r+\lambda}-k\right) .
\end{aligned}
$$

We want to show that $\mathcal{U}^{\prime}\left(M^{-1}\right) \geq 0$ when $c \rightarrow 0$. With this objective in mind, we look for a lower bound for $\bar{U}_{H}-c-\bar{U}_{L}$. Note that

$$
\begin{aligned}
\bar{U}_{H}-c & \geq U_{H}^{F I}\left(\tau_{H}^{F I}\right)-c \\
\bar{U}_{L} & \leq U_{L}^{F B} \equiv \frac{\lambda}{r+\lambda} \frac{1}{r}-\frac{k}{r},
\end{aligned}
$$

where $U_{L}^{F B}$ is the first best payoff. From here, we get that

$$
\bar{U}_{H}-c-\bar{U}_{L} \geq U_{H}^{F I}\left(\tau_{H}^{F I}\right)-c+\frac{k}{r}-\frac{\lambda}{r+\lambda} \frac{1}{r} .
$$

In the limit, when $c \rightarrow 0$ we have that $U_{H}^{F I}\left(\tau_{H}^{F I}\right)-c \rightarrow(1-k) / r=U_{H}^{F B}$. Accordingly, $\lim _{c \rightarrow 0}\left(\bar{U}_{H}-c-\bar{U}_{L}\right) \geq 1 /(r+\lambda)$. Replacing in $\mathcal{U}_{H}^{\prime}\left(M^{-1}\right)$ we get that

$$
\begin{aligned}
\lim _{c \rightarrow 0} \mathcal{U}_{H}^{\prime}\left(M^{-1}\right) & \geq \frac{(M-1)(1-k)}{r}+\left(\bar{U}_{H}-c\right)-\frac{M}{r+\lambda} \\
& =(M-1)\left(\frac{1-k}{r}-\left(\bar{U}_{H}-c\right)\right)+M\left(\bar{U}_{H}-c-\frac{1}{r+\lambda}\right)>0 .
\end{aligned}
$$

This means that for $c$ small enough, $x=M^{-1}$ is optimal and so we have full investment and $q=1-\beta_{H}=0$ being optimal.

Lemma 3. There is $\tilde{c}_{2}>0$ such that for any $c \leq \tilde{c}_{2}$ a solution to equation (16) satisfying $U_{H}^{F I}\left(\tau_{H}^{F I}\right)-c \geq 1 /(r+\lambda)$ exists.

Proof. First, we use the inequality $U_{H}^{F I}\left(\tau_{H}^{F I}\right)-c \geq 1 /(r+\lambda)$ to find a lower bound for $\tau_{H}^{F I}$. 
Using equation (15) we get that $U_{H}^{F I}\left(\tau_{H}^{F I}\right)-c \geq 1 /(r+\lambda)$ if and only if

$$
\tau_{H}^{F I} \geq \underline{\tau} \equiv \frac{1}{r} \log \left(\frac{\lambda /(r+\lambda)-k}{\lambda /(r+\lambda)-k-r c}\right)
$$

For future reference, remember that $\tau_{H}^{F I}$ solves

$$
e^{-(r+\lambda) \tau}\left(U_{H}^{F I}(\tau)-c\right)=\frac{k}{\lambda}
$$

Let

$$
f(\tau) \equiv e^{-(r+\lambda) \tau}\left(U_{H}^{F I}(\tau)-c\right)-\frac{k}{\lambda}=e^{-(r+\lambda) \tau}\left(\frac{1-k}{r}-\frac{1}{1-e^{-r \tau}} c\right)-\frac{k}{\lambda},
$$

so that by definition $f\left(\tau_{H}^{F I}\right)=0$. An equilibrium with full investment satisfying the required properties exists if we can find $\tau \in[\underline{\tau}, \infty)$ such that $f(\tau)=0$. The limit of $f(\tau)$ when $\tau$ goes to infinity is $\lim _{\tau \rightarrow \infty} f(\tau)=-k / \lambda<0$, which means that it is enough to show that $f(\underline{\tau}) \geq 0$. If we evaluate $f(\tau)$ at the lower bound $\underline{\tau}$ we get

$$
f(\underline{\tau})=\left(\frac{\lambda /(r+\lambda)-k-r c}{\lambda /(r+\lambda)-k}\right)^{\frac{r+\lambda}{r}} \frac{1}{r+\lambda}-\frac{k}{\lambda} .
$$

Given the parametric assumption $1 /(r+\lambda)>k / \lambda$, the denominator in the last expression is positive, so the expression is decreasing in $c$ and strictly positive for $c=0$. Hence, $f(\underline{\tau})>0$ if $c \leq \tilde{c}_{2}$ where $\tilde{c}_{2}>0$ is chosen such $f(\underline{\tau})=0$.

Lemma 4. Suppose that $\bar{U}_{H}-c \geq 1 /(r+\lambda)$ then there is $\hat{U}_{L}^{0} \in\left(0, \bar{U}_{H}-c-k / \lambda\right)$ such that

$$
\begin{aligned}
\hat{U}_{L}^{0} & =\int_{0}^{\tau_{L}^{0}} e^{-r t}\left(p_{t}^{L}-k\right) d t+e^{-r \tau_{L}^{0}}\left(p_{\tau_{L}^{0}}^{L}\left(\bar{U}_{H}-c\right)+\left(1-p_{\tau_{L}^{0}}^{L}\right) \hat{U}_{L}^{0}\right) \\
\tau_{L}^{0} & =\frac{1}{r+\lambda} \log \left(\frac{\lambda\left(\bar{U}_{H}-c-\hat{U}_{L}^{0}\right)}{k}\right) .
\end{aligned}
$$

Proof. Let's define the function

$$
G(u)=\int_{0}^{\tau(u)} e^{-r t}\left(p_{t}^{L}-k\right) d t+e^{-r \tau(u)}\left(p_{\tau(u)}^{L}\left(\bar{U}_{H}-c\right)+\left(1-p_{\tau(u)}^{L}\right) u\right)-u
$$

where

$$
\tau(u)=\frac{1}{r+\lambda} \log \left(\frac{\lambda\left(\bar{U}_{H}-c-u\right)}{k}\right)
$$


We need to show that a solution $G(u)=0$ exists on the open interval $\left(0, \bar{U}_{H}-c-k / \lambda\right)$ (the restriction that $\bar{U}_{L}$ is strictly lower than $\bar{U}_{H}-c-k / \lambda$ is required to guarantee that $\left.\tau>0\right)$. Noting that $G\left(\bar{U}_{H}-c-k / \lambda\right)=0$ and $G(0)=\hat{U}_{L}^{1}>0$ we conclude that it is enough to show that $G\left(\bar{U}_{H}-c-k / \lambda-\epsilon\right)<0$ for some small $\epsilon>0$. Because $G(u)$ is continuous, it is sufficient to show that $G^{\prime}\left(\bar{U}_{H}-c-k / \lambda\right)>0$. For convenience, we use the change of variable $x(u) \equiv e^{-r \tau(u)}$ and write

$$
G(u)=\frac{(1-x)(1-k)}{r}+\frac{x^{\alpha}-1}{r+\lambda}+x\left[1-x^{\alpha-1}\right]\left(\bar{U}_{H}-c\right)+x^{\alpha} u-u
$$

where as usual $\alpha \equiv(r+\lambda) / r$. Using the incentive compatibility constraint we can verify that

$$
x^{\prime}(u)=\frac{x(u)}{\alpha\left(\bar{U}_{H}-c-u\right)} .
$$

Differentiating $G(u)$ we get

$$
G^{\prime}(u)=x^{\prime}(u)\left[\bar{U}_{H}-c-\frac{(1-k)}{r}+\frac{x^{\alpha-1}}{r}\right]+2 x^{\alpha}-1
$$

Evaluating at $\hat{u}=U_{H}-c-\frac{k}{\lambda}$ we get

$$
G^{\prime}(\hat{u})=x^{\prime}(\hat{u})\left[\bar{U}_{H}-c+\frac{k}{r}\right]+1>0
$$

As $G(\hat{u})=0$ and $G(0)=\hat{U}_{L}^{1}>0$ there is $\hat{U}_{L}^{0} \in(0, \hat{u})$ such that $G\left(\hat{U}_{L}^{0}\right)=0$.

Lemma 5. There is $\tilde{c}_{3}>0$ such that $\beta_{L}=0$ is optimal for all $c \leq \tilde{c}_{3}$.

Proof. Fix $\theta_{0}=L$.

We want to show that when $c \rightarrow 0, q=1-\beta=1$ is optimal. Consider the firm's payoff after replacing the binding incentive compatibility constraint (recall that in case $\theta_{0}=L$ in the best equilibrium $\tau_{a}=0$, so this expression uses $y=1$.)

$$
\mathcal{U}_{L}(x) \equiv \mathcal{U}_{L}(x, q(x))=\frac{1-k}{r}-\frac{1}{r+\lambda}-\frac{k}{\lambda}-x\left(\frac{1-k}{r}-\left(\bar{U}_{H}-c\right)\right)+\frac{x^{\alpha}}{r+\lambda} .
$$

Note it is convex and the derivative is

$$
\mathcal{U}_{L}^{\prime}(x)=-\left(\frac{1-k}{r}-\left(\bar{U}_{H}-c\right)\right)+\frac{\alpha x^{\alpha-1}}{r+\lambda}
$$


Let $x^{0}=x(q=0)$ and $x^{1}=x(q=1)$ and recall that $x^{1}>x^{0}$. If we replace $x^{0}$ and $\alpha$ we get

$$
\mathcal{U}_{L}^{\prime}\left(x^{0}\right)=-\left(\frac{1-k}{r}-\left(\bar{U}_{H}-c\right)\right)+\frac{1}{r}\left[\frac{k}{\lambda}\left(\bar{U}_{H}-c\right)\right]^{\frac{\alpha-1}{\alpha}} .
$$

It is straightforward to show that $U_{H}^{F I}\left(\tau_{H}^{F I}\right)$ converges to the first best payoff $\frac{1-k}{r}$ as $c$ goes to zero because the frequency of certification remains bounded:

$$
\lim _{c \rightarrow 0} \tau_{H}^{F I}=\frac{1}{r+\lambda} \log \left(\frac{1-k}{r} \frac{\lambda}{k}\right)>0 .
$$

Therefore $\lim _{c \rightarrow 0}\left(\bar{U}_{H}-c-(1-k) / r\right)=0$ which means that $\lim _{c \rightarrow 0} \mathcal{U}_{L}^{\prime}\left(x^{0}\right)>0$. The optimality of $x^{1}$ follows from the convexity of $\mathcal{U}_{L}(x)$.

Lemma 6. It is never optimal for a high quality firm to delay certification at time $\tau_{\theta}^{*}$

Proof. In the case of $\tau_{H}^{*}$ it is straightforward that the firm would not deviate as the deviation payoff is zero (the reputation drops to $p=0$ and even if the firm certifies later, it has to pay $c$ and receive continuation payoff $\underline{U}_{H}=c$ for a net payoff 0 ). The same reasoning applies if $\tau_{L}^{*}$ and $\beta=1$, i.e. if the equilibrium is harsh. The case of $\tau_{L}^{*}$ is a bit different when the equilibrium is lenient, $\beta=0$ because the high quality firm can then deviate to certification at some other on-path time, for example $2 \tau_{L}^{*}$ (the previous reasoning applies if the firm deviates to off-path time). It is sufficient to consider a single-step deviation in which the firm that does not certify at time $\tau_{L}^{*}$ certifies for sure at time $2 \tau_{L}^{*}$. The payoff of such a deviation is

$$
\tilde{U}_{H}=\int_{0}^{\tau_{L}^{*}} e^{-r t}\left(p_{t}^{L}-k\right) d t+e^{-r \tau}\left(\bar{U}_{H}-c\right)
$$

Adding and subtracting $\left(1-p_{\tau_{L}^{*}}^{L}\right) \bar{U}_{L}$ we can write

$$
\begin{aligned}
\tilde{U}_{H} & \left.=\int_{0}^{\tau_{L}^{*}} e^{-r t}\left(p_{t}^{L}-k\right) d t+e^{-r \tau_{L}^{*}}\left(p_{\tau_{L}^{*}}^{L}\left(\bar{U}_{H}-c\right)\right)+\left(\left(1-p_{\tau_{L}^{*}}^{L}\right) \bar{U}_{L}\right)\right)+e^{-r \tau_{L}^{*}}\left(1-p_{\tau_{L}^{*}}^{L}\right)\left(\bar{U}_{H}-c-\bar{U}_{L}\right) \\
& =\bar{U}_{L}+e^{-r \tau_{L}^{*}}\left(1-p_{\tau_{L}^{*}}^{L}\right)\left(\bar{U}_{H}-c-\bar{U}_{L}\right) \\
& =\left(1-e^{-r \tau_{L}^{*}}\left(1-p_{\tau_{L}^{*}}^{L}\right)\right) \bar{U}_{L}+e^{-r \tau_{L}^{*}}\left(1-p_{\tau_{L}^{*}}^{L}\right)\left(\bar{U}_{H}-c\right) \\
& <\bar{U}_{H}-c
\end{aligned}
$$

which means that a high quality firm never has incentives to delay certification at $t=\tau_{L}^{*}$. 
Lemma 7. $\beta_{L}^{*}$ is non-decreasing in $c$

Proof. We show that $q=1-\beta_{L}$ is non-increasing in $c$. Replacing the binding IC constraint, we get that the payoff of a low quality firm given $(x, q)$ (recall $x=e^{-r \tau}$ ) is

$$
\mathcal{U}_{L}(x, q(x))=\frac{1-k}{r}-\frac{1}{r+\lambda}-\frac{k}{\lambda}-x\left(\frac{1-k}{r}-\left(\bar{U}_{H}-c\right)\right)+\frac{x^{\alpha}}{r+\lambda} .
$$

We show that $q$ is non-increasing by using monotone comparative static. Let $\mathcal{U}_{L}(x, q(x), c)$ be the payoff of the low quality firm given by equation (5) as a function of $c$. The cross derivative with respect to $c$ and $x$ is

$$
\frac{\partial^{2}}{\partial x \partial c} \mathcal{U}_{L}(x, q(x), c)=\frac{\partial}{\partial c}\left(\bar{U}_{H}(c)-c\right)=\bar{U}_{H}^{\prime}(c)-1<0 .
$$

Thus, $\mathcal{U}_{L}(x, q(x), c)$ satisfies the single crossing property. Using monotone comparative statics we conclude that $x$ is non-increasing in $c$. Combining the fact that $x=e^{-r \tau}$ and that $\tau$ is higher when $q=0$ we verify that $\tau$ is non-decreasing in $c$. But then the incentive compatibility constraint immediately implies that $q$ is non-increasing in $c$.

\section{Time-Contingent Certification}

As Section II demonstrates, all MPE exhibit poor efficiency properties. On the other hand the best equilibrium analyzed in Section III is very efficient but requires significant coordination between firms and the market. For completeness, here we discuss another class of equilibria, referred to as Time-Contingent Equilibria (TCE): TCE exhibit a simple stationary structure that seems consistent with the way in which many certification programs are organized.

In a TCE, the firm's certification strategy depends on time since last certification rather than reputation. A time-contingent equilibrium is characterized by two numbers: $\tau$, which represents the market belief about the duration of the certificate, and $\tau_{a}$ which represents the time at which the firm starts investing, with $\tau_{a}<\tau$. That is, we consider an equilibrium in which after the firm certifies at time $t_{0}$, the equilibrium prescribes no certification before time $t_{0}+\tau$, and certification with probability one at time $t_{0}+\tau$, if the firm still has high quality. If the firm has low quality, the equilibrium certification strategy is to certify (after $\left.t_{0}+\tau\right)$ as soon as the quality improves. On the other hand, the firm invests, regardless of quality, from time $t_{0}+\tau_{a}$ till the time it certifies again. 
Define $\tau_{c}$ as the largest $\tau$ consistent with an MPE as characterized in section II:

$$
\tau_{c} \equiv \frac{-\log \left(\left[1-\frac{c}{\frac{1}{r+\lambda}-\frac{k}{\lambda}}\right]^{\frac{\lambda}{r+\lambda}}\right)}{\lambda} .
$$

Proposition 1. Define two functions of $\tau$ and $\tau_{a}$ by:

$$
\begin{aligned}
v\left(\tau, \tau_{a}\right) & \equiv \frac{\int_{0}^{\tau} e^{-r t} p_{t} d t-\left(e^{-r \tau_{a}}-e^{-r \tau}\right) \frac{k}{r}+\left(e^{-(r+\lambda) \tau_{a}}-e^{-r \tau_{a}}\right) \frac{k}{\lambda}-c}{1-e^{-r \tau}} \\
g\left(\tau, \tau_{a}\right) & \equiv \frac{k}{\lambda} \frac{r+\lambda}{r} e^{(r+\lambda)\left(\tau-\tau_{a}\right)}-\frac{k}{r} .
\end{aligned}
$$

Let $\tau_{a} \in(0, \tau)$ be a solution of $v\left(\tau, \tau_{a}\right)=g\left(\tau, \tau_{a}\right)$.

If such solution exists, then there is a (time-contingent) equilibrium (TCE) with $a_{t}=$ $\mathbf{1}_{\left\{t>\tau_{a}\right\}}$. In addition, if $v(\tau, 0) \geq g(\tau, 0)$ there exists a TCE with $\tau_{a}=0$. For every $\tau>\tau_{c}$ there exists at least one TCE; and for any equilibrium there is positive investment before $\tau$.

In all TCEs characterized in this proposition the ex-ante equilibrium payoff of the $H$ type firm is $U_{H}(0)-c=v\left(\tau, \tau_{a}\right)$.

Unlike MPE, if $\tau_{a} \in(0, \tau)$, the equilibrium reputation is non-monotone in time between two certification times. The market rationally expects that the firm is shirking right after certification, so reputation goes down right after $t=0$. Yet, as the expiration date of the certificate approaches, the firm starts investing again. The market rationally foresees that and reputation starts going up. Hence, certification happens not when the firm reputation is lowest, but after it rebounds. Generally, our model predicts the following pattern of reputation and certification. If the firm reaches $\tau$ having high quality, certification happens either at the highest reputation (if it started with low quality) or after reputation has recently improved (if it started with high quality). If the firm reaches $\tau$ with low quality, it fails to certify, reputation discontinuously drops, and the firm certifies again after it regains high quality.

For a fixed certificate duration $\tau$, there are sometimes multiple $\tau_{a}$ that are consistent with an equilibrium. This multiplicity is caused by strategic complementarity of reputation and investment: pessimistic beliefs about the firm's investment policy reduce the payoffs from certification and that in turn reduces incentives to invest (and vice-versa). By reducing the return to investment low investment levels may then become a self-fulfilling prophecy. We 
define

$$
\mathcal{E}(\tau) \equiv\left\{t_{a} \in[0, \tau]: v\left(\tau, t_{a}\right) \geq g\left(\tau, t_{a}\right) \text { and }\left(v\left(\tau, t_{a}\right)-g\left(\tau, t_{a}\right)\right) t_{a}=0\right\},
$$

as the set of equilibrium investment thresholds $\tau_{a}$, given duration $\tau$, and we let $\underline{\tau}_{a}=\inf \mathcal{E}(\tau)$ and $\bar{\tau}_{a}=\sup \mathcal{E}(\tau)$ be the lower and higher investment thresholds that can be supported in equilibrium. With this definition, we can further characterize time-contingent equilibria.

Proposition 2. Let $U_{H}\left(0 \mid \tau, \tau_{a}\right)$ be the high-quality firm's ex-ante expected (time-contingent) equilibrium payoff when the certification duration is $\tau$ and the equilibrium investment threshold is $\tau_{a}$. Then

(i) There is some finite $\tau>\tau_{c}$ such that $U_{H}\left(0 \mid \tau, \tau_{a}\right)>V_{H}^{n c}(1)$ for all $\tau_{a} \in \mathcal{E}(\tau)$ where $V_{H}^{n c}(1)$ is the payoff of a firm with reputation $p=1$ from committing to no certification forever.

(ii) $\underline{\tau}_{a}$ and $\bar{\tau}_{a}$ are monotone nondecreasing in $c$ and $k$.

(iii) For any $\tau<\frac{1}{r+\lambda} \log \left(\frac{\lambda}{r+\lambda} \frac{1}{k}\right)$ there is $\bar{c}>0$ such that $\underline{\tau}_{a}=0$ for all $c \leq \bar{c}$.

(iv) Let $\underline{U}_{H}(0 \mid \tau):=U_{H}\left(0 \mid \tau, \underline{\tau}_{a}\right)$ and $\bar{U}_{H}(0 \mid \tau):=U_{H}\left(0 \mid \tau, \bar{\tau}_{a}\right)$ be the ex-ante expected payoff in the equilibrium with minimum and maximum investment threshold, respectively. Then, $\max _{\tau \geq \tau_{c}} \underline{U}_{H}(0 \mid \tau)$ and $\max _{\tau \geq \tau_{c}} \bar{U}_{H}(0 \mid \tau)$ are decreasing in $c$ and $k$.

Under MPE the high-quality firm would be better off by committing to no-certification (for the proof, see working paper version). By contrast, Proposition 2 shows that there exists a duration of certificates $\tau$ that generates better payoffs than a commitment to no certification, no matter what equilibrium $\tau_{a}$ the firm and the market coordinate on. Therefore, if the duration is chosen optimally, we can overcome the paradoxical result that certification does not promote investment and hurts the firm, under MPE.

The optimal duration $\tau$ is determined by the following trade off. As $\tau$ changes there are two equilibrium effects: First, a longer $\tau$ reduces expected certification costs, which increases the firm payoffs and incentives to invest in quality, close to $\tau$. Second, longer $\tau$ means that the firm has to wait a long time till recertification hence the firm may choose to shirk right after certification. This trade off is such that the optimum is always interior: neither no certification nor highly frequent recertification -as under MPE- are optimal.

A lower $c$, holding the frequency of certification constant, increases the payoffs of the high quality firm, increasing incentives to invest in quality, which means the firm starts investing 
sooner (and rational expectations by the market reinforce this effect). Finally, Proposition 2 states that firms with high quality benefit from lower certification costs in time-contingent equilibria with optimal $\tau$. That resolves another paradox of the belief-contingent equilibria discussed in Section II. ${ }^{1}$

Overall, the best TCE leads to better outcomes than any MPE. Recall that MPE can only trigger certification when the firm's reputation has decreased sufficiently. Hence, some spells of shirking must always be part of an MPE. This is not always true for TCE: by disconnecting certification times from the firm's reputation, TCE are often able to implement higher levels of investment and lower frequency of certification. It is important to note here that in a different model where, where even under full effort high quality is not an absorbing state, reputation would drift even under full effort. In that case, the best MPE and TCE would exhibit similar properties. To see this, consider the case when TCE implements full effort at all times. Since reputation is a deterministic function of time within certification cycles, if a TCE prescribed certification at time $\tau$, we could construct an MPE prescribing certification when reputation reaches $p_{\tau}$.

We conclude by contrasting TCE with the best equilibrium. In what sense is TCE restrictive relative to the best equilibrium? Relative to TCE, the best equilibrium reduces the ability of low reputation firms to certify quality as soon as quality improves, thereby reducing expected certification expenses - without affecting the firm's expected reputation. Indeed, TCE prescribes that a low quality firm that failed to certify its quality during the last scheduled review will certify it as soon as it improves; this possibility leads to excessive certification expenses. Second, the best equilibrium improves incentives because it's able to make stronger threats against non-certifying firms: when the best equilibrium is harsh, a firm that fails to certify at time $\tau$ is essentially shut down, because it loses its ability to certify in the future. Under TCE, by contrast, a low reputation firm that failed to certify can always restart afresh when quality improves. This access to "forgiveness" that characterizes TCE sometimes weakens the firm's investment incentives.

\footnotetext{
${ }^{1}$ This last comparison is somewhat complicated since even for the optimal $\tau$ there may be multiple timecontingent equilibria with different $\tau_{a}$, so we compare equilibria with the lowest and the highest selections of the equilibrium investment.
} 


\section{Proofs of results in Appendix C on Time-Contingent Equilibria}

Because between certifications the firm reputation is a deterministic function of time, then for every Markov perfect equilibrium we characterized in the previous section, in which the high quality firm certifies in intervals of length $\tau_{c}$, there exists an outcome-equivalent timecontingent equilibrium where $\tau=\tau_{c}$. To focus on equilibria with more investment than in the previous section, we restrict attention to equilibria with $\tau$ larger than $\tau_{c}$, where we define $\tau_{c} \equiv \frac{-\log p_{c}^{-}}{\lambda}$ as the amount of time that elapses before reputation reaches the certification threshold $p_{c}^{-}$in the most-efficient belief-contingent equilibrium characterized in Proposition 1. Moreover, we focus on equilibria in which the low-quality invests when reputation is at the lowest and maintain the assumption that $c<\frac{1}{r+\lambda}-\frac{k}{\lambda}$.

\section{Proof of Proposition 1}

Proof. To analyze these time-contingent equilibria, we first consider the firm's investment incentives for a fixed $\tau$. Since the equilibrium is stationary (on path), without loss of generality we reset the time clock to $t_{0}=0$ when the firm certifies high quality. To avoid confusion, since the state variable is different in this section than in the previous one, we introduce new notation: we denote the value function and value of quality as $U_{\theta}(t)$ and $\bar{D}(t)$, where $t$ is the time since last certification and we write the investment strategy as $a_{t}$.

On the equilibrium path, the continuation value satisfies a HJB equation similar to the one in the Markov case

$$
\begin{aligned}
& 0=\max _{a \in[0,1]} p_{t}-a k+\dot{U}_{L}(t)+\lambda a \bar{D}(t)-r U_{L}(t) \\
& 0=\max \left\{\max _{a \in[0,1]} p_{t}-a k+\dot{U}_{H}(t)-\lambda(1-a) \bar{D}(t)-r U_{H}(t),\right. \\
& \left.U_{H}^{d}-c-U_{H}(t)\right\},
\end{aligned}
$$

where $U_{H}^{d}$ is the continuation value if the firm certifies early. As we mentioned before, we can consider the punishment continuation equilibrium with $U_{H}^{d}-c=0$; this means, that no early certification is incentive compatible as long as $U_{H}(t) \geq 0$. Looking at the investment strategy, analogously to our reasoning in the previous section, at time $t<\tau$ the firm's 
investment incentives depend on

$$
\bar{D}(t)=e^{-(r+\lambda)(\tau-t)} \bar{D}(\tau) .
$$

In any (time-contingent) equilibrium, the firm invests at time $t$ if and only if $\lambda \bar{D}(t) \geq k$, so the optimal investment strategy is also time-contingent. Equation (12) implies that $\bar{D}(t)$ is increasing, so that investment must be a non-decreasing function of time. In other words, the firm's investment strategy defined as a function of time must take the form $a_{t}=\mathbf{1}_{t>\tau_{a}}$ for some threshold $\tau_{a} \leq \tau$, where $\tau_{a}=\tau$ indicates that the firm never invests. ${ }^{2}$

We compute the firm's continuation value $U_{\theta}$ in several steps: first, we compute the continuation value at expiration, namely at $t=\tau$, then we determine $\tau_{a}$ as a function of continuation payoffs, then work backwards to obtain the continuation value for $t<\tau$, and finally solve a fixed-point problem to determine $\tau_{a}$ and the continuation payoffs.

Since we are looking at equilibria in which the low-quality firm invests at time $t=\tau$ (and thereafter until the realization of the first positive shock) its continuation value is

$$
U_{L}(\tau)=\frac{\lambda\left(U_{H}(0)-c\right)-k}{r+\lambda}
$$

which means that the value of quality at time $t$ is

$$
\bar{D}(t)=e^{-(r+\lambda)(\tau-t)} \bar{D}(\tau)=e^{-(r+\lambda)(\tau-t)} \frac{r\left(U_{H}(0)-c\right)+k}{r+\lambda} .
$$

This allows us to pin down the firm's investment strategy, namely the time $\tau_{a}$ at which the firm starts investing. The firm is indifferent between investing and not at $t=\tau_{a}$ if the return to investment is zero, i.e., if $\tau_{a}$ satisfies:

$$
e^{-(r+\lambda)\left(\tau-\tau_{a}\right)} \frac{r\left(U_{H}(0)-c\right)+k}{r+\lambda}=\frac{k}{\lambda} .
$$

Solving for $\tau_{a}$ yields

$$
\tau_{a}=\tau+\frac{1}{r+\lambda} \log \left(\frac{r+\lambda}{\lambda} \frac{k}{r\left(U_{H}(0)-c\right)+k}\right) .
$$

\footnotetext{
${ }^{2}$ Optimal investment strategy at $t=\tau_{a}$ is not uniquely determined, but since the firm reaches $\tau_{a}$ over a zero measure of all the times, this has no impact on total payoffs. Hence, when we describe equilibria, we ignore this indeterminacy.
} 
Of course, equation (15) is valid for $\tau_{a} \in[0, \tau]$. A straightforward computation shows that $\tau_{a}>0$ if and only if the return to investment is negative at $t=0$, namely $\bar{D}(0)<k / \lambda$. If this condition does not hold, then the equilibrium entails first-best investment, $\tau_{a}=0$. On the other hand, $\tau_{a} \leq \tau$ if and only if the return to investment at time $\tau$ is strictly positive or, $\lambda\left(U_{H}(0)-c\right)-k>0$. In words, the firm is willing to invest prior to $\tau$ if the return to investment at time $\tau$ is strictly positive.

The next step is to compute the firm value during the investment interval, $t \in\left[\tau_{a}, \tau\right)$. Because there is no certification during this interval, the firm value consists of two components: the present value of the cash flows earned through $[t, \tau)$ and the value of the firm at time $\tau$ net of the certification cost that will be incurred at that time:

$$
U_{H}(t)=\int_{t}^{\tau} e^{-r(s-t)}\left(p_{s}-k\right) d s+e^{-r(\tau-t)}\left(U_{H}(0)-c\right),
$$

where $p_{t}$ evolves according to $\dot{p}_{t}=\lambda\left(1-p_{t}\right)$, (since $a_{t}=1$ in that interval). Using $p_{a}$ as the initial belief in the interval $\left[\tau_{a}, \tau\right)$, we obtain

$$
p_{t}=1-e^{-\lambda\left(t-\tau_{a}\right)}\left(1-p_{a}\right)
$$

Using the definition of $\bar{D}(\cdot)$ and equation (13), we get that the low-quality firm value for $t \in\left[\tau_{a}, \tau\right)$ is

$$
U_{L}(t)=U_{H}(t)-e^{-(r+\lambda)(\tau-t)} \bar{D}(\tau)
$$

The final step in the construction of the value functions requires that we consider the interval $t \in\left[0, \tau_{a}\right]$, when the firm is not investing. Given that there is no investment during this interval, reputation is $p_{t}=e^{-\lambda t}$ so the continuation values are

$$
\begin{aligned}
U_{L}(t) & =\int_{t}^{\tau_{a}} e^{-r(s-t)} p_{s} d s+e^{-r\left(\tau_{a}-t\right)} U_{L}\left(\tau_{a}\right) \\
U_{H}(t) & =\int_{t}^{\tau_{a}} e^{-r(s-t)} p_{s} d s+e^{-r\left(\tau_{a}-t\right)} U_{L}\left(\tau_{a}\right)+e^{-(r+\lambda)\left(\tau_{a}-t\right)} \bar{D}\left(\tau_{a}\right) .
\end{aligned}
$$

Notice the asymmetry between the two states: because in this interval the firm is not investing, it can experience a negative shock in the high state but no shocks in the low state.

We can now pin down the investment threshold $\tau_{a}$ using equation (18), along with (15) and the optimality condition $\bar{D}\left(\tau_{a}\right)=k / \lambda$. At the same time we can pin down the equilibrium payoffs as a solution to a fixed-point problem and establish existence of equilibria. 
Using equation (18), together with the optimality condition $\bar{D}\left(\tau_{a}\right)=k / \lambda$ we get

$$
\begin{aligned}
U_{H}(0) & =\int_{t}^{\tau_{a}} e^{-r(s-t)} p_{s} d s+e^{-r\left(\tau_{a}-t\right)}\left[U_{H}\left(\tau_{a}\right)-\bar{D}\left(\tau_{a}\right)\right]+e^{-(r+\lambda)\left(\tau_{a}-t\right)} \frac{k}{\lambda} \\
& =\int_{t}^{\tau_{a}} e^{-r(s-t)} p_{s} d s+e^{-r\left(\tau_{a}-t\right)} U_{H}\left(\tau_{a}\right)+\left(e^{-(r+\lambda)\left(\tau_{a}-t\right)}-e^{-r\left(\tau_{a}-t\right)}\right) \frac{k}{\lambda} .
\end{aligned}
$$

Replacing $U_{H}\left(\tau_{a}\right)$ and evaluation at $t=t_{0}$ we get

$$
U_{H}(0)=\int_{0}^{\tau_{a}} e^{-r s} p_{s} d s+\int_{\tau_{a}}^{\tau} e^{-r s}\left(p_{s}-k\right) d s+e^{-r \tau}\left(U_{H}(0)-c\right)+\left(e^{-(r+\lambda) \tau_{a}}-e^{-r \tau_{a}}\right) \frac{k}{\lambda} .
$$

Computing the integral of the price in equation (20) yields

$$
\begin{aligned}
h\left(\tau, \tau_{a}\right) \equiv \int_{0}^{\tau} e^{-r s} p_{s} d s & =\frac{1}{r+\lambda}-\frac{e^{-(r+\lambda) \tau_{a}}}{r+\lambda}+\frac{e^{-r \tau_{a}}-e^{-r \tau}}{r} \\
& \frac{e^{-(r+\lambda) \tau_{a}}-e^{-(r+\lambda) \tau}}{r+\lambda}+\frac{e^{-(r+\lambda) \tau+\lambda \tau_{a}}-e^{-r \tau_{a}}}{r+\lambda} \\
& =\frac{1}{r+\lambda}+\frac{e^{-r \tau_{a}}-e^{-r \tau}}{r}-\frac{e^{-(r+\lambda) \tau}}{r+\lambda}+e^{-r \tau_{a}} \frac{e^{-(r+\lambda)\left(\tau-\tau_{a}\right)}-1}{r+\lambda}
\end{aligned}
$$

Replacing in and (20) and defining $v \equiv U_{H}(0)-c$ we get (8). Computing $g$ as the inverse of (15) we get

$$
g\left(\tau, \tau_{a}\right) \equiv \frac{k}{\lambda} \frac{r+\lambda}{r} e^{(r+\lambda)\left(\tau-\tau_{a}\right)}-\frac{k}{r}
$$

In equilibrium, if $\tau_{a}>0$, it must be the case that

$$
g\left(\tau, \tau_{a}\right)=v\left(\tau, \tau_{a}\right)
$$

Finally, suppose that $v(\tau, 0) \geq g(\tau, 0)$. By definition, this means

$$
\bar{D}(0)=e^{-(r+\lambda) \tau} \frac{r v+k}{r+\lambda} \geq \frac{k}{\lambda}
$$

so $\tau_{a}=0$ is optimal for the seller.

The next step is to show that for any $\tau>\tau_{c}$ an equilibrium exists and $\tau_{a}<\tau$. By continuity, it suffices to show that $v(\tau, \tau)>g(\tau, \tau)$. First, we have that $g(\tau, \tau)$ is

$$
g(\tau, \tau)=\frac{k}{\lambda} \frac{r+\lambda}{r}-\frac{k}{r}=\frac{k}{\lambda}
$$


Evaluating the RHS at $\tau_{a}=\tau$ we get

$$
v(\tau, \tau)=\frac{\frac{1}{r+\lambda}-\frac{e^{-(r+\lambda) \tau}}{r+\lambda}+\left(e^{-(r+\lambda) \tau}-e^{-r \tau}\right) \frac{k}{\lambda}-c}{1-e^{-r \tau}}
$$

If $c<\frac{1}{r+\lambda}-\frac{k}{\lambda}$ then $\lim _{\tau \rightarrow \infty} v(\tau, \tau)>\frac{k}{\lambda}$. Next, evaluating the limit at $\tau_{c}$ we obtain

$$
v\left(\tau_{c}, \tau_{c}\right)=\frac{k}{\lambda}
$$

As

$$
\frac{d}{d \tau} v(\tau, \tau)=\frac{r e^{-r \tau}}{1-e^{-r \tau}}\left(\frac{k}{\lambda}-v(\tau, \tau)\right)+\frac{(r+\lambda) e^{-(r+\lambda) \tau}}{1-e^{-r \tau}}\left(\frac{1}{r+\lambda}-\frac{k}{\lambda}\right)
$$

we get that $v(\tau, \tau)=k / \lambda$ implies $\frac{d}{d \tau} v(\tau, \tau)>0$. Hence, $v\left(\tau_{c}, \tau_{c}\right)=\frac{k}{\lambda}$ implies that $v(\tau, \tau)>\frac{k}{\lambda}$ for all $\tau>\tau_{c}$.

The only step left is to show that $U_{H}(t) \geq 0$, all $t \in[0, \tau]$, so $U_{H}(t) \geq U_{H}^{d}-c$ (where $U_{H}^{d}$ is the worst equilibrium in Proposition 1). Looking for a proof by contradiction, suppose that there is $\hat{t} \in\left[\tau_{a}, \tau\right]$ such that $U_{L}(\hat{t})<0$. Because $U_{L}(\tau)>0$, by continuity, there is $\tilde{t}>\hat{t}$ such that $U_{L}(\tilde{t})=0$. Using the HJB equation for $U_{L}$ we get that

$$
\dot{U}_{L}(\tilde{t})=-p_{\tilde{t}}-(\lambda \bar{D}(\tilde{t})-k)<0,
$$

which means that $U_{L}(\tilde{t}+\epsilon)<0$; hence, $U_{L}(t)$ can never cross zero and $U_{L}(\tau)<0$, which give us the desired contradiction. Using the fact that $U_{H}(t) \geq U_{L}(t)$ we conclude that $U_{H}(t) \geq 0$ for all $t \in\left[\tau_{a}, \tau\right]$. The final step is to look at the interval $\left[0, \tau_{a}\right]$. For any $t \leq \tau_{a}$, the low type continuation value satisfies

$$
U_{L}(t)=\int_{t}^{\tau_{a}} e^{-r(s-t)} p_{s} d s+e^{-r\left(\tau_{a}-t\right)} U_{L}\left(\tau_{a}\right)
$$

We have already shown that $U_{L}\left(\tau_{a}\right) \geq 0$, which means that $U_{L}(t)$ above is greater then zero. Hence, we conclude that $U_{H}(t) \geq 0$, all $t \in\left[0, \tau_{a}\right]$. 


\section{Proof of Proposition 2}

\section{Proposition 2(i)}

First we note that for any $\tau_{a}^{0}, \tau_{a}^{1} \in \mathcal{E}(\tau)$ it is the case that $\tau_{a}^{1}>\tau_{a}^{0}$ implies $U\left(0 \mid \tau, \tau_{a}^{0}\right)>$ $U\left(0 \mid \tau, \tau_{a}^{1}\right)$. This follows from the fact that for any $\tau_{a}>0$

$$
U\left(0 \mid \tau, \tau_{a}\right)=g\left(\tau, \tau_{a}\right)+c
$$

is decreasing in $\tau_{a}$ and when $\tau_{a}=0$ we have $U(0 \mid \tau, 0)>g(\tau, 0)+c$. Hence, it is sufficient to show that there is $\tau$ such that $U\left(0 \mid \tau, \bar{\tau}_{a}\right)>V^{n c}\left(p_{0}\right)$. We complete the proof with two lemmas. In Lemma 8 we show that there is a benefit setting $\tau>\tau_{c}$, while in Lemma 9 we show that $\tau=\infty$ is not optimal.

Lemma 8. Suppose that $\frac{1}{r+\lambda}-\frac{k}{\lambda}>c$ then $\left.\frac{d}{d \tau} v\left(\tau, \tau_{a}(\tau)\right)\right|_{\tau=\tau_{c}}>0$.

Proof. Suppose that $\tau_{a}>0$. In this case, we have that

$$
\begin{aligned}
\frac{d}{d \tau} v\left(\tau, \tau_{a}(\tau)\right) & =v_{\tau}\left(\tau, \tau_{a}\right)+v_{\tau_{a}}\left(\tau, \tau_{a}\right) \tau_{a}^{\prime}(\tau) \\
\tau_{a}^{\prime}(\tau) & =-\frac{v_{\tau}\left(\tau, \tau_{a}\right)-g_{\tau}\left(\tau, \tau_{a}\right)}{v_{\tau_{a}}\left(\tau, \tau_{a}\right)-g_{\tau_{a}}\left(\tau, \tau_{a}\right)}
\end{aligned}
$$

Where,

$$
\begin{aligned}
g_{\tau}\left(\tau, \tau_{a}\right) & =\frac{k}{\lambda} \frac{(r+\lambda)^{2}}{r} e^{(r+\lambda)\left(\tau-\tau_{a}\right)}=(r+\lambda) g\left(\tau, \tau_{a}\right)+(r+\lambda) \frac{k}{r} \\
g_{\tau_{a}}\left(\tau, \tau_{a}\right) & =-\frac{k}{\lambda} \frac{(r+\lambda)^{2}}{r} e^{(r+\lambda)\left(\tau-\tau_{a}\right)}=-g_{\tau}\left(\tau, \tau_{a}\right) \\
v_{\tau}\left(\tau, \tau_{a}\right) & =\frac{e^{-r \tau}}{1-e^{-r \tau}}\left(\frac{1-k}{r}-v\right)+\frac{e^{-(r+\lambda) \tau}-e^{-r \tau-\lambda\left(\tau-\tau_{a}\right)}}{1-e^{-r \tau}} \\
v_{\tau_{a}}\left(\tau, \tau_{a}\right) & =\frac{e^{-r \tau_{a}}\left(k\left(1-e^{-\lambda \tau_{a}}\right)(r+\lambda)^{2}+\lambda^{2}\left(e^{-(r+\lambda)\left(\tau-\tau_{a}\right)}-1\right)\right)}{\lambda(r+\lambda)\left(1-e^{-r \tau}\right)}
\end{aligned}
$$


Evaluating at $\tau=\tau_{a}=\tau_{c}$ and using $v\left(\tau_{c}, \tau_{c}\right)=g\left(\tau_{c}, \tau_{c}\right)=k / \lambda$ we get

$$
\begin{aligned}
g_{\tau}\left(\tau_{c}, \tau_{c}\right) & =(r+\lambda) \frac{k}{\lambda}+(r+\lambda) \frac{k}{r}>0 \\
g_{\tau_{a}}\left(\tau_{c}, \tau_{c}\right) & =-(r+\lambda) \frac{k}{\lambda}-(r+\lambda) \frac{k}{r}<0 \\
v_{\tau}\left(\tau_{c}, \tau_{c}\right) & =\frac{(r+\lambda) e^{-r \tau_{c}}}{1-e^{-r \tau_{c}}}\left(\frac{p_{c}}{r+\lambda}-\frac{k}{\lambda}\right) \\
v_{\tau_{a}}\left(\tau_{c}, \tau_{c}\right) & =\frac{k}{\lambda} \frac{\left(1-e^{-\lambda \tau_{c}}\right)(r+\lambda)}{e^{r \tau_{c}}-1}=\frac{(r+\lambda) e^{-r \tau_{c}}}{1-e^{-r \tau_{c}}} \frac{k}{\lambda}\left(1-p_{c}\right)>0
\end{aligned}
$$

Noting that we can write

$$
v_{\tau}\left(\tau_{c}, \tau_{c}\right)=\frac{(r+\lambda) p_{c} e^{-r \tau_{c}}}{1-e^{-r \tau_{c}}}\left(\frac{1}{r+\lambda}-\frac{k}{\lambda}\right)-v_{\tau_{a}}\left(\tau_{c}, \tau_{c}\right) .
$$

Replacing in the equation for $\frac{d}{d \tau} v\left(\tau, \tau_{a}(\tau)\right)$

$$
\left.\frac{d}{d \tau} v\left(\tau, \tau_{a}(\tau)\right)\right|_{\tau=\tau_{c}}=\frac{(r+\lambda) p_{c} e^{-r \tau_{c}}}{1-e^{-r \tau_{c}}}\left(\frac{1}{r+\lambda}-\frac{k}{\lambda}\right)+v_{\tau_{a}}\left(\tau_{c}, \tau_{c}\right)\left(\tau_{a}^{\prime}\left(\tau_{c}\right)-1\right)
$$

Moreover, we have

$$
\begin{aligned}
\tau_{a}^{\prime}\left(\tau_{c}\right)-1 & =-\frac{v_{\tau}\left(\tau_{c}, \tau_{c}\right)+v_{\tau_{a}}\left(\tau_{c}, \tau_{c}\right)}{v_{\tau_{a}}\left(\tau_{c}, \tau_{c}\right)-g_{\tau_{a}}\left(\tau_{c}, \tau_{c}\right)} \\
& =-\frac{\frac{(r+\lambda) p_{c} e^{-r \tau_{c}}}{1-e^{-r \tau_{c}}}\left(\frac{1}{r+\lambda}-\frac{k}{\lambda}\right)}{v_{\tau_{a}}\left(\tau_{c}, \tau_{c}\right)-g_{\tau_{a}}\left(\tau_{c}, \tau_{c}\right)}
\end{aligned}
$$

which means that

$$
\left.\frac{d}{d \tau} v\left(\tau, \tau_{a}(\tau)\right)\right|_{\tau=\tau_{c}}=\frac{(r+\lambda) p_{c} e^{-r \tau_{c}}}{1-e^{-r \tau_{c}}}\left(1-\frac{v_{\tau_{a}}\left(\tau_{c}, \tau_{c}\right)}{v_{\tau_{a}}\left(\tau_{c}, \tau_{c}\right)-g_{\tau_{a}}\left(\tau_{c}, \tau_{c}\right)}\right)>0
$$

Finally, suppose that $\tau_{a}=0$. In this case, we have that

$$
\frac{d}{d \tau} v\left(\tau, \tau_{a}(\tau)\right)=v_{\tau}\left(\tau, \tau_{a}\right)=\frac{e^{-r \tau}}{1-e^{-r \tau}}\left(\frac{1-k}{r}-v\right)>0
$$

Where the last inequality follows from the fact that $(1-k) / r$ is the payoff in the first best and so it is necessarily grater than $v$.

Lemma 9. Suppose that $\frac{1}{r+\lambda}-\frac{k}{\lambda}>c$ then there is $\tau$ such that $U_{H}\left(0 \mid \tau, \bar{\tau}_{a}\right)>V_{H}^{n c}\left(p_{0}\right)$. 
Proof. We start showing that $\lim _{\tau \rightarrow \infty}\left(\bar{\tau}_{a}(\tau)-\tau\right)=0$ and $\lim _{\tau \rightarrow \infty} \bar{\tau}_{a}^{\prime}(\tau)=1$. This implies that

$$
\lim _{\tau \rightarrow \infty} \frac{d}{d \tau} v\left(\tau, \bar{\tau}_{a}\right)=\lim _{\tau \rightarrow \infty} v_{\tau}\left(\tau, \bar{\tau}_{a}\right)+v_{\tau_{a}}\left(\tau, \bar{\tau}_{a}\right) \bar{\tau}_{a}^{\prime}(\tau)=v_{\tau}\left(\tau, \bar{\tau}_{a}\right)+v_{\tau_{a}}\left(\tau, \bar{\tau}_{a}\right)=\lim _{\tau \rightarrow \infty} \frac{d}{d \tau} v(\tau, \tau)
$$

The final step is to show that $\frac{d}{d \tau} v(\tau, \tau)<0$ for $\tau$ arbitrarily large.

Step 1: $\lim _{\tau \rightarrow \infty}\left(\bar{\tau}_{a}(\tau)-\tau\right)=0$ and $\lim _{\tau \rightarrow \infty} \bar{\tau}_{a}^{\prime}(\tau)=1$.

Given that $v\left(\tau, \tau_{a}\right)$ is bounded above and $g(\tau, 0) \rightarrow \infty$ as $\tau \rightarrow \infty$ it must be the case that $\bar{\tau}_{a}>0$ for $\tau$ sufficiently large. Defining $x \equiv \exp (-r \tau)$ and $y \equiv \exp \left(-r \tau_{a}\right)$ we can write the equilibrium condition for $\tau_{a}$ in terms of $x$ and $y$ as

$$
\frac{k}{\lambda} \frac{r+\lambda}{r} y^{1+\frac{\lambda}{r}}=x^{1+\frac{\lambda}{r}}\left[\frac{k}{r}+\frac{\left.\left(\frac{1-x^{1+\frac{\lambda}{r}}}{r+\lambda}+\frac{y-x}{r}+y^{r} \frac{\left(\frac{x}{y}\right)^{1+\frac{\lambda}{r}}-1}{r+\lambda}\right)-\frac{(y-x) k}{r}+\frac{\left(y^{1+\frac{\lambda}{r}}-y\right) k}{\lambda}-c\right]}{1-x}\right] .
$$

By direct inspection of the above equation we conclude that the limit when $\tau \rightarrow \infty$ which corresponds to the limit when $x \rightarrow 0$ is given by

$$
\lim _{x \rightarrow 0}(y-x)=0
$$

Moreover, replacing $x$ and $y$ into equation (21)

$$
\bar{\tau}_{a}^{\prime}=-\frac{\frac{x}{1-x}\left(\frac{1-k}{r}-v\right)+\frac{x^{1+\frac{\lambda}{r}}-\left(\frac{x}{y}\right)^{1+\frac{\lambda}{r}} y}{(1-x)}-\frac{k}{\lambda} \frac{(r+\lambda)^{2}}{r}\left(\frac{y}{x}\right)^{1+\frac{\lambda}{r}}}{\frac{y\left(k\left(1-y^{\frac{\lambda}{r}}\right)(r+\lambda)^{2}+\lambda^{2}\left(\left(\frac{x}{y}\right)^{1+\frac{\lambda}{r}}-1\right)\right)}{\lambda(r+\lambda)(1-x)}+\frac{k}{\lambda} \frac{(r+\lambda)^{2}}{r}\left(\frac{y}{x}\right)^{\left(1+\frac{\lambda}{r}\right)}} .
$$

and taking the limit when $x \rightarrow 0$ we get

$$
\lim _{x \rightarrow 0} \bar{\tau}_{a}^{\prime}=-\frac{-\frac{k}{\lambda} \frac{(r+\lambda)^{2}}{r}}{-\frac{k}{\lambda} \frac{(r+\lambda)^{2}}{r}}=1 .
$$

Step 2: We show that $\lim _{x \rightarrow 0} \frac{d v(\tau(x), \tau(x))}{d x}>0$. This, along with Step 1, implies that the 
optimal $\tau$ is interior. Substituting $x=e^{-r \tau}$ into $v(\tau, \tau)$ yields

$$
v(\tau(x), \tau(x))=\frac{\frac{1}{r+\lambda}-\frac{x^{1+\frac{\lambda}{r}}}{r+\lambda}+\left(x^{1+\frac{\lambda}{r}}-x\right) \frac{k}{\lambda}-c}{1-x} .
$$

Differentiating $v(\tau(x), \tau(x))$

$$
\frac{d v(\tau(x), \tau(x))}{d x}=\frac{-\frac{x^{\frac{\lambda}{r}}}{r}+\left(\frac{r+\lambda}{r} x^{\frac{\lambda}{r}}-1\right) \frac{k}{\lambda}}{1-x}+\frac{\frac{1}{r+\lambda}-\frac{x^{1+\frac{\lambda}{r}}}{r+\lambda}+\left(x^{1+\frac{\lambda}{r}}-x\right) \frac{k}{\lambda}-c}{(1-x)^{2}}
$$

and evaluating at $x=0$ we get

$$
\left.\frac{d v(\tau(x), \tau(x))}{d x}\right|_{x=0}=\frac{1}{r+\lambda}-\frac{k}{\lambda}-c>0 .
$$

By Step 1 we have that

$$
\lim _{x \rightarrow 0} \frac{d v(\tau(x), \tau(x))}{d x}=\lim _{x \rightarrow 0} \frac{d}{d x} v\left(\tau(x), \bar{\tau}_{a}(\tau(x))\right)
$$

Hence, we have that for $\tau$ arbitrarily large

$$
\frac{d v\left(\tau, \bar{\tau}_{a}(\tau)\right)}{d \tau}=\frac{d v\left(\tau(x), \bar{\tau}_{a}(\tau(x))\right)}{d x} \frac{d x}{d \tau}=-r e^{-r \tau} \frac{d v\left(\tau(x), \bar{\tau}_{a}(\tau(x))\right)}{d x}<0
$$

so the $\tau$ that maximizes $v\left(\tau, \bar{\tau}_{a}(\tau)\right)$ is interior.

\section{Proposition 2(ii)}

Let's define $f\left(t_{a}, c\right):=g\left(\tau, t_{a}\right)-v\left(\tau, t_{a}\right)$. The derivative of $f(\cdot, c)$ with respect to $c$ is $\frac{1}{1-e^{-r \tau}}>0$ so we have that $f\left(t_{a}, c_{1}\right) \geq f\left(t_{a}, c_{0}\right)$ for any $c_{1}>c_{0}$. Accordingly, Lemma 1 in ? implies that $\underline{\tau}_{a}\left(c_{1}\right)=\inf \left\{t_{a} \in[0, \tau]: f\left(t_{a}, c_{1}\right) \leq 0\right\} \geq \inf \left\{t_{a} \in[0, \tau]: f\left(t_{a}, c_{0}\right) \leq 0\right\}=\underline{\tau}_{a}\left(c_{0}\right)$ and $\bar{\tau}_{a}\left(c_{1}\right)=\sup \left\{t_{a} \in[0, \tau]: f\left(t_{a}, c_{1}\right) \geq 0\right\} \geq \sup \left\{t_{a} \in[0, \tau]: f\left(t_{a}, c_{0}\right) \geq 0\right\}=\bar{\tau}_{a}\left(c_{0}\right)$. Similarly, differentiating $g$ and $v$ with respect to $k$ we get that $g\left(\tau, t_{a}\right)-v\left(\tau, t_{a}\right)$ is increasing in $k$, which means that $\tau_{a}$ and $\bar{\tau}_{a}$ are decreasing in $k$ as well.

\section{Proposition 2(iii)}

Evaluating $v\left(\tau, \tau_{a}\right)$ and $g\left(\tau, \tau_{a}\right)$ at $\tau_{a}=0$ we get the sufficient condition for full effort 


$$
(r+\lambda)\left(1-e^{-r \tau}\right)\left(\frac{1}{r+\lambda}-\frac{k}{\lambda} e^{(r+\lambda) \tau}\right) \geq r c .
$$

Clearly, for any fixed $\tau$ we can find $\bar{c}>0$ such that the previous condition is satisfied for all $c \leq \bar{c}$ if and only if

$$
\frac{1}{r+\lambda}-\frac{k}{\lambda} e^{(r+\lambda) \tau}>0
$$

for some $\tau>0$. Te LHS is decreasing in $\tau$ and by assumption $\frac{1}{r+\lambda}>\frac{k}{\lambda}$; hence, the previous inequality is satisfied for all $\tau<\frac{1}{r+\lambda} \log \left(\frac{\lambda}{r+\lambda} \frac{1}{k}\right)$.

\section{Proposition 2(iv)}

We prove the result only for $\sup _{\tau \geq \tau_{c}} \underline{U}_{H}(0 \mid \tau)$ as the proof for $\sup _{\tau \geq \tau_{c}} \bar{U}_{H}(0 \mid \tau)$ is analogous. Let's consider $c_{1}>c_{0}$; from Proposition 2(ii) we have that $\underline{\tau}_{a}\left(c_{1}\right) \geq \underline{\tau}_{a}\left(c_{0}\right)$; which means that it suffices to show that $U_{H}\left(1 \mid \tau, \tau_{a}, c\right)$ is decreasing in $\tau_{a}$. Fix $\tau$ and consider the case with $\underline{\tau}_{a}\left(c_{0}\right)>0$. Using equation $(9)$, we have that

$$
U_{H}\left(0 \mid \tau, \bar{\tau}_{a}(\tau, c), c\right)-c=g\left(\tau, \bar{\tau}_{a}(\tau, c)\right)
$$

Hence, the firm's ex-ante profit given an investment threshold $t_{a}$ is

$$
\begin{aligned}
U_{H}\left(0 \mid \tau, t_{a}, c\right) & =h\left(\tau, t_{a}\right)-\left(e^{-r t_{a}}-e^{-r \tau}\right) \frac{k}{r}-e^{-r \tau}\left(1-p_{\tau}\right) \frac{k}{r+\lambda} \\
& +e^{-r \tau}\left(\frac{\lambda}{r+\lambda}+\frac{r}{r+\lambda} p_{\tau}\right)\left(U_{H}\left(0 \mid \tau, t_{a}, c_{0}\right)-c_{0}\right) \\
& =h\left(\tau, t_{a}\right)-\left(e^{-r t_{a}}-e^{-r \tau}\right) \frac{k}{r}-e^{-r \tau}\left(1-p_{\tau}\right) \frac{k}{r+\lambda} \\
& +e^{-r \tau}\left(\frac{\lambda}{r+\lambda}+\frac{r}{r+\lambda} p_{\tau}\right) g\left(\tau, t_{a}\right),
\end{aligned}
$$

where the function $h$ is defined in Proposition 2 and in the second equation we have replaced equation (26). The derivative with respect to $t_{a}$ is

$$
\frac{e^{-(r+\lambda) t_{a}}\left(r\left(k r(r+\lambda)-\lambda^{2}\right) e^{\lambda t_{a}}-k(r+\lambda)^{3} e^{\lambda \tau}-k r(\lambda+r)^{2}+\lambda^{2} r e^{-(r+\lambda)\left(\tau-t_{a}\right)}\right)}{\lambda r(\lambda+r)}
$$

The sign of the previous expression is determined by the sign of the denominator which is

$$
-k(r+\lambda)\left((r+\lambda)^{2} e^{\lambda \tau}-r^{2} e^{\lambda t_{a}}+r(\lambda+r)\right)-\lambda^{2} r\left(e^{\lambda t_{a}}-e^{-(r+\lambda)\left(\tau-t_{a}\right)}\right)<0 .
$$


Hence, we have that $\frac{\partial}{\partial t_{a}} U_{H}\left(0 \mid \tau, t_{a}, c\right)<0$. Next, we consider the case with $\tau_{a}\left(c_{0}\right)=0$. If $\tau_{a}\left(c_{1}\right)>0$ then $U_{H}\left(0 \mid \tau, \tau_{a}\left(c_{0}\right), c_{0}\right)$ is strictly greater than (28) so the previous argument for the case with $\tau_{a}\left(c_{0}\right)$ applies and $U_{H}\left(0 \mid \tau, \tau_{a}\left(c_{0}\right), c_{0}\right)>U_{H}\left(0 \mid \tau, \tau_{a}\left(c_{1}\right), c_{1}\right)$. Finally, the case with $\tau_{a}\left(c_{0}\right)=\tau_{a}\left(c_{1}\right)=0$ is trivial as both policies have the same investment, the same certification and one has a lower cost. Repeating the same argument in the case of $k$ we get that $U_{H}\left(0 \mid \tau, \tau_{a}\right)$ is also decreasing in $k$. 